\title{
EE-LEACH: development of energy-efficient LEACH Protocol for data gathering in WSN
}

\author{
Gopi Saminathan Arumugam ${ }^{{ }^{*}}$ and Thirumurugan Ponnuchamy ${ }^{2^{*}}$
}

\begin{abstract}
A wireless sensor network (WSN) consists of a huge number of sensor nodes that are inadequate in energy, storage and processing power. One of the major tasks of the sensor nodes is the collection of data and forwarding the gathered data to the base station (BS). Hence, the network lifetime becomes the major criteria for effective design of the data gathering schemes in WSN. In this paper, an energy-efficient LEACH (EE-LEACH) Protocol for data gathering is introduced. It offers an energy-efficient routing in WSN based on the effective data ensemble and optimal clustering. In this system, a cluster head is elected for each clusters to minimize the energy dissipation of the sensor nodes and to optimize the resource utilization. The energy-efficient routing can be obtained by nodes which have the maximum residual energy. Hence, the highest residual energy nodes are selected to forward the data to BS. It helps to provide better packet delivery ratio with lesser energy utilization. The experimental results shows that the proposed EE-LEACH yields better performance than the existing energy-balanced routing protocol (EBRP) and LEACH Protocol in terms of better packet delivery ratio, lesser end-to-end delay and energy consumption. It is obviously proves that the proposed EE-LEACH can improve the network lifetime.
\end{abstract}

Keywords: Clustering; Cluster head; Energy-efficient routing; Low energy adaptive clustering hierarchy (LEACH); Wireless sensor networks

\section{Introduction}

A wireless sensor network (WSN) consists of a large number of small-sensor nodes used to monitor areas, collect and report data to the base station (BS). Due to the accomplishment in low-power digital circuit and wireless transmission, most of the applications of WSN are implemented and used in military applications, object tracking, habitat monitoring. A typical WSN is composed of a huge number of sensor nodes, which are randomly disseminated over the network. The signals are picked by all types of sensors and the data acquiring unit, processing and transmitting them into a node called sink node. The sink node requests for the sensor information by forwarding a query throughout the network. When the node discovers the data matching the query, the response message is routed back to the sink node. The energy conservation of the network can be

\footnotetext{
* Correspondence: gopiswamiphd2013@hotmail.com; thirumurugan2014@hotmail.com

'Department of Electronics and Communication Engineering, SSMIET, Dindigul 624002, India

${ }^{2}$ Department of Electronics and Communication Engineering, PSNA College of Engineering and Technology, Dindigul 624622, India
}

minimized by allowing the porting of the nodes called cluster heads. The data gathered from the nodes are aggregated and compressed by the cluster heads. After that, the aggregated data is forwarded to the BS, but it has some problems. The major problem is energy consumption and it is concentrated on the cluster heads. In order to resolve this issue, the cluster routing is used to distribute the energy consumption with the cluster heads.

Data gathering is an efficient method for conserving energy in sensor networks. The major purpose of data gathering is to remove the redundant data and save transmission energy [1-3]. A data-gathering algorithm includes some aggregation methods to minimize the data traffic. It reduces the number of message exchange among the nodes and BS. The performance of data gathering in WSN can be characterized based on the rate at which the sensing information can be gathered and transmitted to the BS (or sink node). In particular, the speculative measure to capture the demerits of collection processing in WSN is the capacity for many-to-one data collection. Data-gathering capacity reflects how

\section{穴}


efficient the sink can gather sensing data from all sensors under the presence of interference. Performing the data-gathering function over $\mathrm{CH}$ still causes significant energy wastage. In case of homogenous sensor networks, $\mathrm{CH}$ will soon die and re-clustering needs to be initiated. It causes higher energy consumption.

In this paper, an energy-efficient LEACH Protocol is introduced. The proposed method focuses on defining an energy-efficient routing based on low energy adaptive clustering hierarchy $(\mathrm{LEACH})$ clustering and optimal cluster head $(\mathrm{CH})$ selection. The Gaussian distribution model is incorporated for the node deployment. The data are forwarded from the different sources to the BS based on the energy-efficient routing strategy. The rest of the paper is organized as follows. Section 2 presents a description about the previous research which is relevant to energy-efficient data-gathering approaches. Section 3 describes the proposed energy-efficient LEACH (EELEACH) Protocol. Section 4 presents the results and discussion. Section 5 discusses about the conclusions and future work.

\section{Related works}

Many research activities have been carried out on the area of energy-efficient data gathering in WSN, since the basic task of the WSN is to effectively collect the data with lesser resource consumption. Most of the datagathering algorithms are aimed to minimize the energy consumption problem.

LEACH is a hierarchical protocol, in which the node details are handled by $\mathrm{CHs}[4,5]$. The $\mathrm{CHs}$ gather the data and compress them and forward to the base station (sink). Every node uses the stochastic algorithm to find out the $\mathrm{CH}$. Figure 1 shows the architecture of the standard LEACH Protocol. During the setup phase, each node creates a random number between 0 and 1 . If the random number is smaller than the threshold value, then the node becomes a $\mathrm{CH}$ for the present round. The threshold value is calculated based on the following equation:

$$
K(s)=\left(\frac{p}{1-p\left(r \bmod \frac{1}{p}\right)} \text { if } s \in G\right)
$$

Here, $p$ is the desired percentage of $\mathrm{CH}, r$ denotes the count of present round, and $G$ is the group of sensor nodes that are not $\mathrm{CHs}$ in the previous $1 / p$ rounds. The schematic structure of the proposed LEACH Protocol is shown in Figure 1.

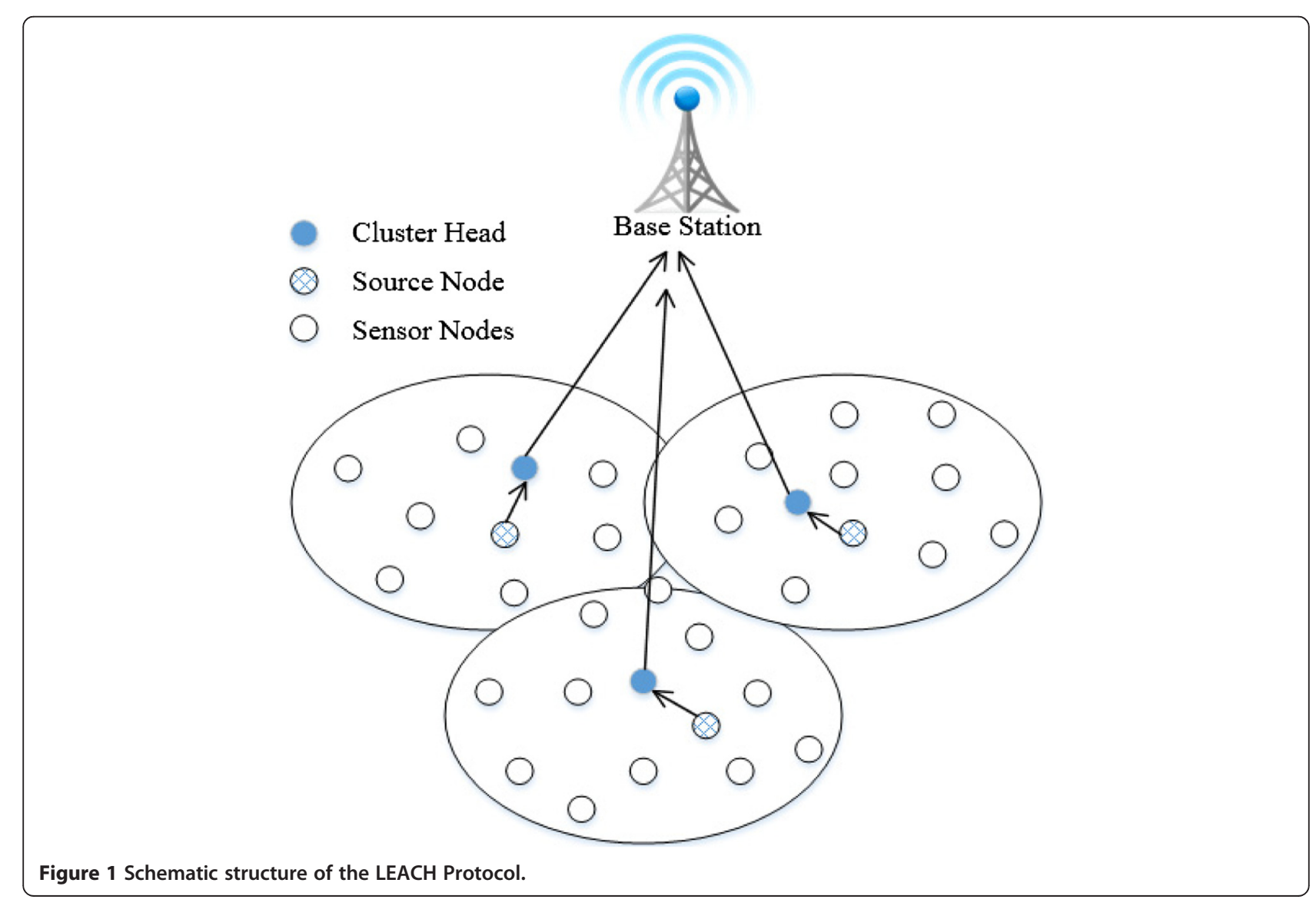


The drawbacks of this protocol are as follows:

- A sensor node is selected as the $\mathrm{CH}$-using distributed probabilistic approach, whereas the non- cluster nodes calculate which cluster to join based on the signal strength. This approach assures lower message overhead, but cannot assure that $\mathrm{CHs}$ are uniformly distributed over the network. The entire network is divided into clusters and load imbalance among the CHs may lead to minimum network lifetime.

- It is assumed that all nodes are isomorphic and all nodes have similar amount of energy capacity in each election round. Such a supposition is impractical in most application circumstances. Hence, LEACH should be enhanced to report for node heterogeneity.

- LEACH involves source nodes to send data to $\mathrm{CHs}$ directly. However, if the $\mathrm{CH}$ is extremely far away from the source nodes, they might expend excessive energy in data transmission. Further, LEACH requires $\mathrm{CHs}$ to transfer their aggregated data to the sink node over a single-hop link. Nevertheless, single-hop transmission may be quite costly when the sink appears far away from the $\mathrm{CH}$.

- LEACH also holds an assumption that all sensor nodes have sufficient power to reach the sink node if necessary, which might be resistant for energyconstrained sensor nodes.

Yao et al. introduced an energy-efficient, delay-aware and lifetime-balancing data collection protocol for WSN. This method proposed both a centralized heuristic to make the algorithm scalable for huge-scale network operations [6]. Han et al. suggested an algorithm for data communication in duty cycle WSNs. The authors in [7] survey the research problem to reveal the insights into problems of duty cycled WSNs. Liu et al. presented a compressed data aggregation for energy-efficient WSNs. The authors in [8] aimed to reduce the energy consumption with the help of joint routing and compressed aggregation. The optimal solution was characterized to this optimization problem, which has proven the NPcompleteness. Moreover, a mixed-integer programming formulation with the greedy heuristic approach was proposed for both the optimal and near-optimal aggregation trees to be obtained.

Chilamkurti et al. formulated a cross layer support for energy-efficient routing in WSNs. It was extended with the dynamic source routing (DSR) to increase its routing energy efficiency by reducing the frequency of recomputed routes. It enables DSR to initiate a route discovery only when link failure occurs [9]. Guiyi et al. presented a prediction-based data aggregation in WSN. It combines the concept of grey model and Kalman filter by integrating the quality of a grey model in quick modeling with the benefit of the Kalman filter in processing data series noise [10]. Liu et al. designed a compressive data collection for WSN. Liu et al. [11] adopt a power-law decaying-data model verified by real datasets and proposed a random projection-based estimation algorithm for this data model. This method needs only fewer measurements, which reduces the number of sensor readings for each measurement. It minimizes the energy consumption without much control and computation overheads.

Zhu et al. presented an energy-efficient data-gathering algorithm to improve the network lifetime. A data gathering sequence (DGS) was used to eliminate the mutual transmission and loop transmission between the nodes. Also, a mathematical programming model was used to compute the minimal remaining energy and total consumption of energy among the nodes. Later, a genetic algorithm was applied to identify the optimal solution for the programming problem [1]. Dhilip et al. proposed an energy-efficient clustering and data aggregation protocol for the heterogeneous WSN. This protocol was designed based on the ideas of data aggregation on energyefficient cluster-based routing. The cluster head election technique was used, and the routing path was selected based upon the sum of residual energy used for data transmission [12]. Wei et al. introduced an energyefficient clustering solution for WSN. A distributed clustering algorithm was used to calculate the appropriate cluster size. It was determined based on the hop distance from the source to sink. An energy-efficient multi hop data-gathering protocol was applied to validate the effectiveness of the cluster and calculate the end-to-end delay [13].

Xiang et al. proposed an energy-efficient clustering algorithm to maximize the lifetime. The clustering algorithm with optimum parameters was used to reduce the energy conservation among the nodes. An analytical clustering with one hop distance and clustering angle was used. Moreover, the optimal one hop distance and clustering angle were conveyed by reducing the energy consumption between inter and intracluster. For each cluster, the continuous procedure gets repeated until the optimum number of clusters were obtained. It reduces the frequency of updating the cluster head and significantly reduces energy to establish a cluster head [14]. Fengyuan et al. formulated an energy-balanced routing protocol for data gathering. Enhanced mechanisms were used to identify and eliminate the loops [15]. Dervis et al. utilized an artificial bee colony algorithm for energy-efficient clustering. The artificial bee colony algorithm was used to prolong the lifetime of the sensor nodes and the network [16]. Yuea et al. discussed about the balanced cluster-based data aggregation algorithm. 
The sensor network was divided into rectangular grids. For each grid, the cluster head was elected to manage the nodes and balance the load among the sensors [17].

Rout et al. introduced an adaptive data aggregation mechanism based on network coding. Here, the group of nodes act as network coder nodes and the remaining nodes were used for relaying purpose. The network coder nodes were sometimes used as aggregation points based on the measure of the data correlation [18]. Hui et al. formulated an exact and heuristic algorithm for data gathering. The data-gathering algorithm was based on the cluster-based approach. A mixed-integer linearprogramming model was used to calculate the BS and $\mathrm{CH}$ position and also the data flow in the network area. This method utilizes both the energy and position of the sensor for selecting the $\mathrm{CH}$. Hence, it avoids the highest energy consumption. The benders decomposition was incorporated into the upper bound heuristics algorithm [19]. Mathapati et al. designed an energyefficient reliable data aggregation approach. Clustering approach was used to group the node into clusters. A coordinate node was elected to monitor the cluster nodes. The $\mathrm{CH}$ was elected based upon the energy level and the distance from the node to the coordinate node. The messages were gathered by $\mathrm{CH}$ and forwarded to the BS [20].

Sheu et al. discussed an efficient path planning and data-gathering protocols in WSN. Here, an infrastructurebased data-gathering protocol and distributed datagathering protocols were introduced to plan the data-gathering route for a BS. Also, k-hop approach was used to limit the number of hops for routing the data to a mobile sink [21]. Ebrahimi et al. proposed a compressive data-gathering approach based on the random projection. The compressive data gathering improves the energy efficiency among the sensors. The random projection was integrated with the compressive data gathering in order to enhance the energy consumption and load throughout the network. A minimum spanning tree projection was used to randomly select the projection nodes [22]. Min et al. proposed an approximate data-gathering approach called EDGES. It utilizes the temporal and spatial correlations. The multiple-model Kalman filter was used to predict the future values based on the previous single-sensor reading. A redistribution model was used to distribute the energy consumption of $\mathrm{CH}$ based on the spatial correlation [23]. Jin et al. presented an adaptive datagathering mechanism based on compressive sensing. Here, an autoregressive model was used in the reconstruction of the sensed data. The local correlation among the sensed data was included, and hence, local adaptive sparsity was obtained. The sink had recovered the data based on the successive reconstructions.
The measurements were modified based on the variation of the sensed data. An abnormal readings detection and identification methods were incorporated based on the combinational sparsity reconstruction [24].

Song et al. proposed a biology-based algorithm to minimal exposure problem (MEP) of WSNs. It explores the biological model of physarum to formulate a biology-inspired optimization algorithm. The MEP with the related models was formulated, and then, it was converted into a Steiner problem through discretizing the monitoring domain [25]. Liu et al. presented a physarum optimization method. It was a biology-inspired algorithm for the Steiner tree problem. A cellular computing model was exploited to solve the Steiner problem [26]. Li et al. designed an opportunistic feeding and routing protocol for reliable multicasting with network coding [27]. Zeng et al. proposed a directional routing and scheduling for green vehicular delay tolerant networks. It solves the routing and scheduling problem as a learning process through geographic routing and flow control toward the optimal direction. A hybrid method with forwarding and replication was presented to speed up the learning process according to the traffic pattern [28].

\section{Proposed method - EE-LEACH: energy-efficient LEACH Protocol}

An efficient-energy-aware routing protocol is mandatory for data gathering. All the sensor nodes have similar significance and equal capabilities. This motivates the need for improving the lifetime of the sensor nodes and sensor network. The objective of the proposed EE-LEACH Protocol is to reduce the energy consumption and increase the network longevity. Here, Gaussian distribution model is used for effective coverage of the sensing network area. Also, conditional probability theorem is used for node aggregation. The flow of the EE-LEACH Protocol is depicted in Figure 2.

\subsection{Topology construction}

Consider a sensor network of $N$ nodes and base station $B S$ is distributed over an area. The position of the sensor nodes and the base station are known beforehand. Let us consider a network in 2D plane with $N$ nodes and it is deployed on the sensing field by 2D Gaussian distribution. It is described as:

$$
f(m, n)=\frac{1}{2 \pi \sigma_{m} \sigma_{n}} e^{-\left(\frac{\left(m-m_{i}\right)^{2}}{2 \sigma_{m}^{2}}+\frac{\left(n-n_{i}\right)^{2}}{2 \sigma_{n}^{2}}\right)}
$$

Where, $\left(m_{i} n_{i}\right)$ denotes the deployment point, $\sigma_{m}$ and $\sigma_{n}$ are the standard deviation for $m$ and $n$ dimensions, respectively. The deployment point is taken as the 


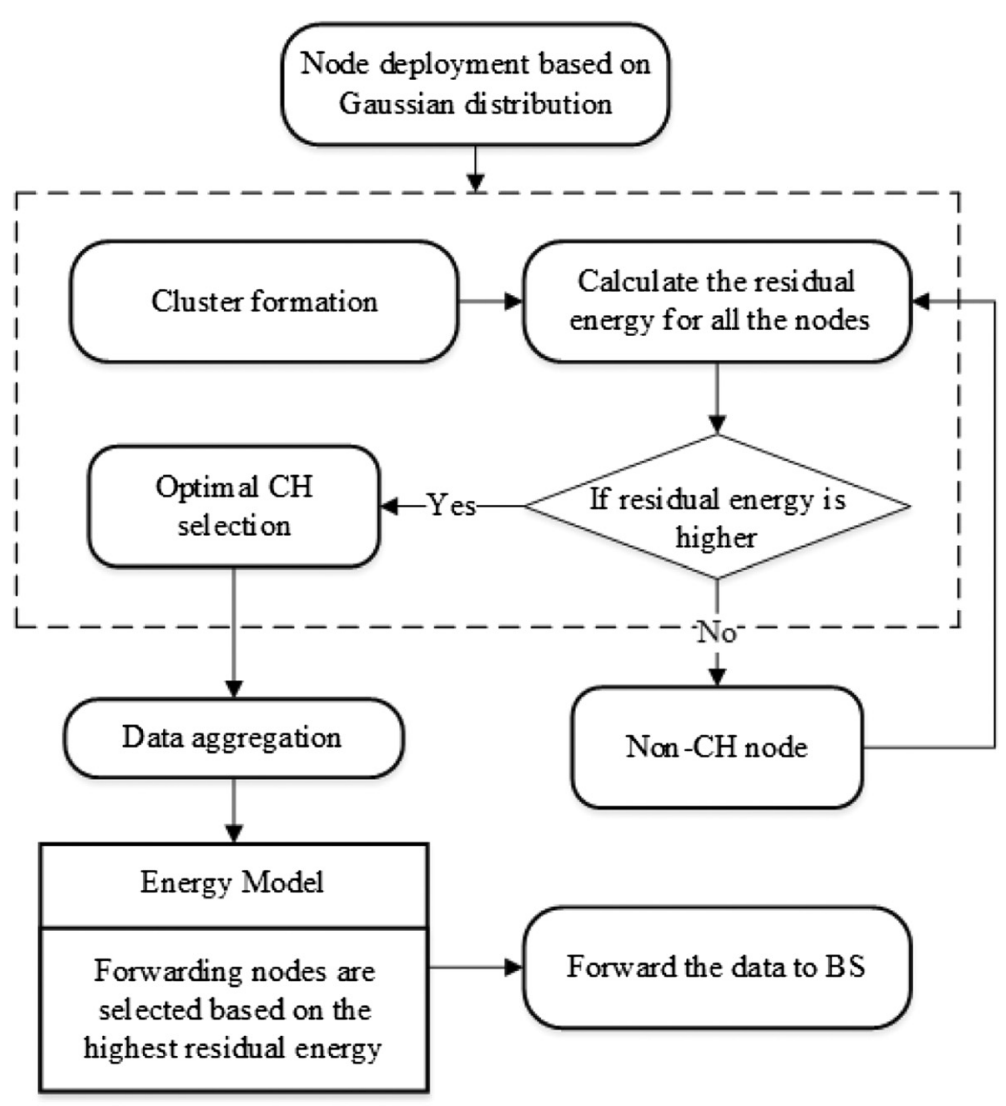

Figure 2 Flow of the proposed EE-LEACH Protocol.

central point of the disk. $\left(m_{i}=n_{i}=0\right)$. The Gaussian distribution is given as:

$$
f(m, n)=\frac{1}{2 \pi \sigma_{m} \sigma_{n}} e^{-\left(\frac{m^{2}}{2 \sigma_{m}^{2}}+\frac{n^{2}}{2 \sigma_{n}^{2}}\right)}
$$

Each node senses the traffic pattern about its data, and a BS is responsible for gathering the data periodically.

\subsection{Optimal cluster formulation}

The formation of clusters in sensor networks highly depends on the time taken to receive the neighbor node message and the residual energy. The protocol is divided into rounds, and each round is triggered to find out the optimal $\mathrm{CH}$. The clusters are formed based on the following steps:

\subsubsection{Step 1: neighbor information retrieval}

The neighbor node information are sensed by broadcasting the beacon messages throughout the network.

\subsubsection{Step 2: perform sorting algorithm}

The sorting algorithm is performed to retrieve the list of all neighbor nodes about its hop distance. The list is sorted into descending order.

\subsubsection{Step 3: candidate for cluster}

When its two- hop neighbor node is not enclosed, analyze all the members of stage 2 one-by-one and crown any one two-hop neighbor for being as a candidate for the cluster.

\subsubsection{Step 4: calculate the residual energy of neighbor nodes}

Finally, the sorting algorithm is executed based on the residual energy of the neighbor nodes.

\section{3 $\mathrm{CH}$ selection}

The computations are based on the following simplifications: assume that the intracluster transmission stage is long. Hence all the data nodes can forward the data to their $\mathrm{CH}$ and intercluster transmission is long enough; hence, all $\mathrm{CH}$ having data can forward their data to the $\mathrm{BS}$. The $\mathrm{CH}$ needs to perform the data aggregation and 
compression before forwarding the data to the BS. The optimal probability of a sensor node is elected as a $\mathrm{CH}$ based on the function of spatial density. The clustering approach is optimal in the sense that overall energy utilization is minimum. Such optimal clustering is greatly dependent on the energy model.

If the signal-to-noise ratio forwards the $M$ bit message across $l$ distance, then the energy expanded can be defined as:

$$
E_{T}(M, l)= \begin{cases}M * E_{g}+M * \varepsilon_{f} * l^{2} & \text { if } l \leq l_{0} \\ M * E_{g}+M * \varepsilon_{h} * l^{4} & \text { if } l \geq l_{0}\end{cases}
$$

Where, $E_{g}$ denotes the energy dissipated per bit, $\varepsilon_{f}$ and $\varepsilon_{h}$ are the transmission ability and $l$ is the distance from the sender to BS. To retrieve the $M$ bit message, the system expends

$$
E_{R}=M \cdot E_{g} .
$$

Moreover, the energy dissipated in the $\mathrm{CH}$ is defined as:

$$
\begin{aligned}
E_{C H}= & \left(\frac{n}{k}-1\right) * M * E_{g}+\frac{n}{k} * M * E_{D}+M * E_{g} \\
& +M * \varepsilon_{f} * l_{B S}^{2}
\end{aligned}
$$

Here, $k$ is defined as the number of clusters, $E_{D}$ denotes the processing cost for a bit report to the BS, and $l_{B S}$ represents the average distance between the $\mathrm{CH}$ and BS. The energy dissipated for normal nodes is:

$$
E_{\text {normal }}=M * E_{g}+M * \varepsilon_{f} * l_{C H}^{2}
$$

Where $l_{\mathrm{CH}}$ denotes the average distance between the normal sensor nodes and the $\mathrm{CH}$. Consider that the nodes are uniformly distributed, which is defined as:

$$
\begin{aligned}
l_{C H}^{2} & =\int_{0}^{X_{\max }} \int_{0}^{Y_{\max }}\left(\left(x^{2}+y^{2}\right) * \rho(x, y)\right) d x d y \\
& =\frac{A^{2}}{2 \pi k}
\end{aligned}
$$

$\rho(x, y)$ is the node distribution. The overall energy dissipated in the network is given as follows:

$$
E_{O}=M *\left(2 * n * E_{g}+n * E_{D}+\left(k * l_{B S}^{2}+n * l_{C H}^{2}\right)\right)
$$

The optimal probability for a normal node to become a $\mathrm{CH}$ can be calculated based on the following equation:

$$
P_{\text {Otm }}=\frac{k_{\text {Otm }}}{n}
$$

\subsubsection{Benefits for optimal $\mathrm{CH}$ election}

The selection of $\mathrm{CH}$ nodes in the sensor networks can provide the following three benefits:
Prolonging network lifetime - In the form of heterogeneous networks,

1. the average energy utilization for transmitting the data from the sensor node to the BS will be much lesser than the energy utilized for homogenous networks.

2. Improving reliability of data forwarding - It is generally known that the links tend to be low reliability. Each hop significantly minimizes the packet delivery rate. In heterogeneous nodes, there will be lesser hops between the nodes and the BS. Hence, the heterogeneous sensor networks can achieve a much better packet delivery ratio than the homogenous networks.

3. Decreasing latency for data transmission Computational heterogeneity can minimize the latency in immediate nodes. The heterogeneity among the links can minimize the waiting time in the forwarding queue. Choosing lesser hops among the nodes to BS will reduce the forwarding latency.

\subsection{Data aggregation based on data ensemble}

A cluster of nodes is replaced with a single node without changing the underlying joint deployment of the network. During node aggregation, the data ensemble process also takes place. It is essential to identify the macro node for data aggregation. Henceforth, the procedure includes two steps:

1. Path definition and

2. Pair of combinable nodes.

The conditional probability is applied for the node aggregation process. The conditional probability of the macro node should be equal to the product of all the component nodes. Consider the following network in Figure 3:

If the nodes $B, C$, and $D$ are combined into a macro node $M$, then the condition probability is given by:

$$
P_{C}(M \mid A)=P(B, C, D \mid A)=P(B \mid A) P(C \mid A) P(D \mid B, C)
$$

The conditional probability of a macro node's successor is equivalent to the conditional probability of the successor given all the component sensor nodes in the macro node, except the nodes that are not linked directly to the successor node. Here, $E$ is the successor and the above statement is given as:

$$
P_{C}(E \mid M)=P(E \mid B, C, D)
$$

By aggregating the sensor nodes using the above equations, the data also gathered for transmission. 


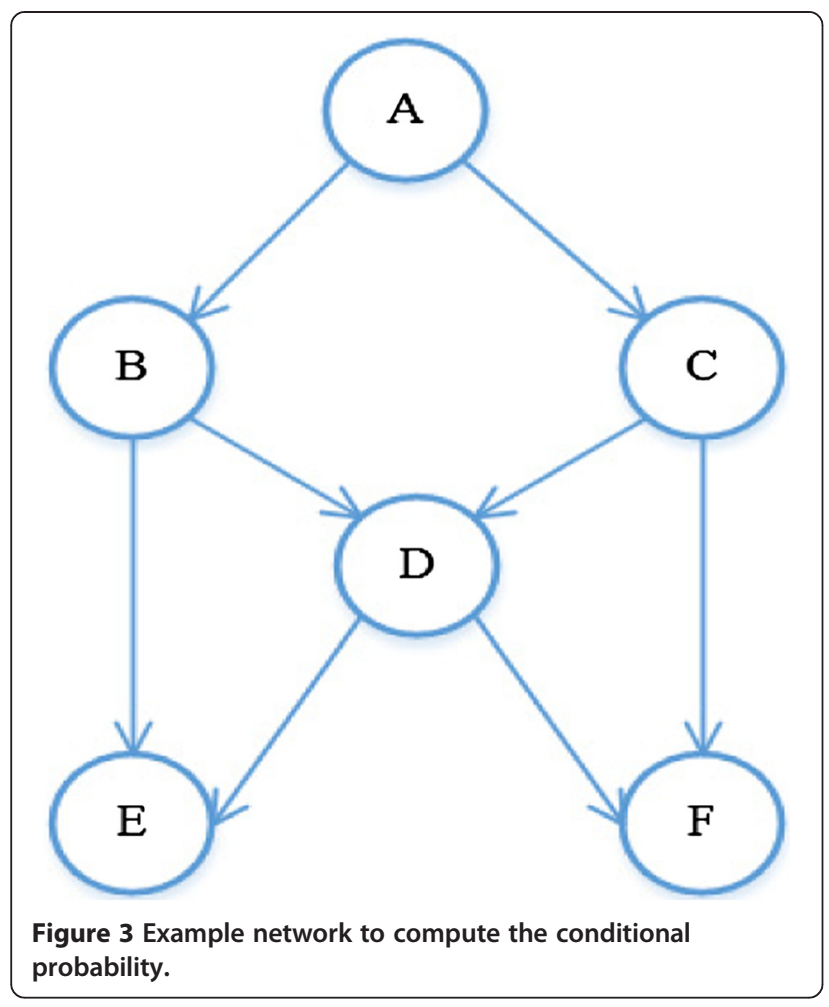

\subsection{Energy-efficient data routing}

After gathering the data from different clusters, the $\mathrm{CHs}$ need to forward the data to the BS. Hence, the forwarding nodes are selected based on the highest residual energy among the nodes. The nodes which are having the highest energy are selected to forward the data to the BS. It helps to improve the packet delivery ratio from the packet loss.

\section{Performance analysis}

In this section the performance of the proposed EELEACH Protocol is experimented and compared with the existing energy-balanced routing protocol (EBRP) [15] and LEACH [4] Protocol. There are 100 nodes deployed in the $100 \times 100 \mathrm{~m}^{2}$. The efficiency of the proposed system is evaluated based on the following criteria: throughput, variance of energy, energy consumption for data aggregation, average energy utilization, remaining residual energy, endto-end delay and packet delivery ratio. Table 1 presents the simulation parameters. The sensor nodes are randomly

Table 1 Simulation parameters

\begin{tabular}{ll}
\hline Parameters & Values \\
\hline Total number of sensor nodes & 100 \\
Network size & $100 \times 100 \mathrm{~m}$ \\
Node distribution & Random \\
Initial energy & $2 \mathrm{~J}$ \\
Base station location & $(110,45)$ \\
\hline
\end{tabular}

distributed with the initial node energy as $2 \mathrm{~J}$. It is considered that the position of the BS is $(110,45)$. If the node's energy is equal or less than 0 , then the node is considered as the dead node and it is removed from the data aggregation.

Throughput is the total number of successfully received data in a unit time. The maximum stable throughput is the maximum number of traffic per unit time and it is measured in bits/second. It can be injected into the network from all the source nodes. Moreover, the size of the queue at any sensor node is restricted. Generally, it is considered that all the nodes generate an equivalent quantity of network traffic. Figure 4 shows the throughput analysis of the proposed EE-LEACH with the existing EBRP and LEACH Protocol. From the results, it proves that the proposed system can successfully do the data aggregation from the sources to BS.

Figure 5 depicts the relationship between the variance of energy with the varying simulation time for EELEACH with the existing EBRP and LEACH Protocols. The result shows that the proposed system lesser variance than the existing protocol.

Figure 6 shows the result for average energy utilization of the two protocols. It is visually proven that the average energy utilization is lesser than the existing EBRP and LEACH Protocols. End-to-end delay is the total amount of time the system takes to aggregate the data from the source to BS.

$$
\text { EtoE delay }=\frac{\sum(\text { arrive time-send time })}{\sum \text { number of connections }}
$$

Figure 7 shows the end-to-end delay between the EELEACH with EBRP and LEACH Protocols. It shows that the proposed protocol takes lesser time to aggregate and

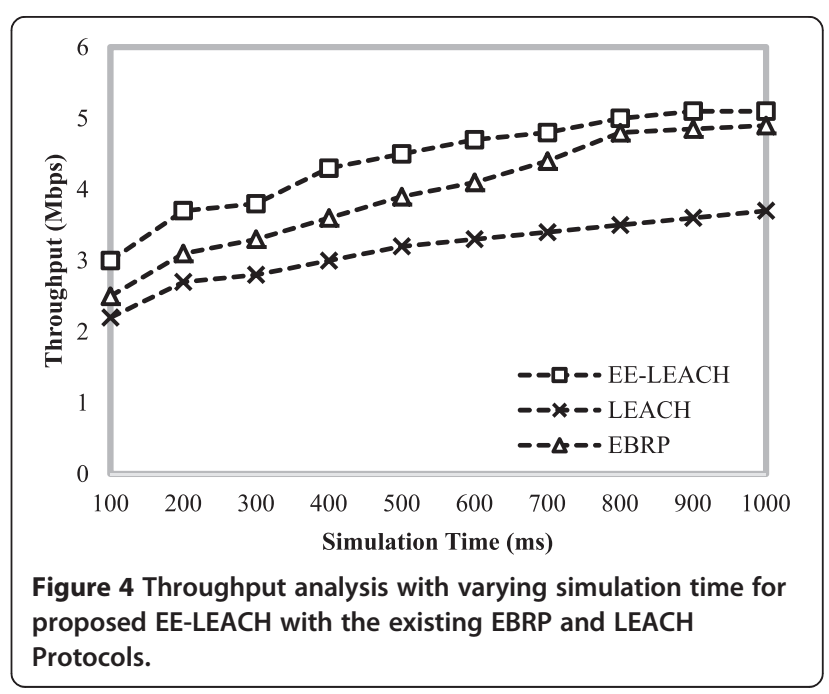




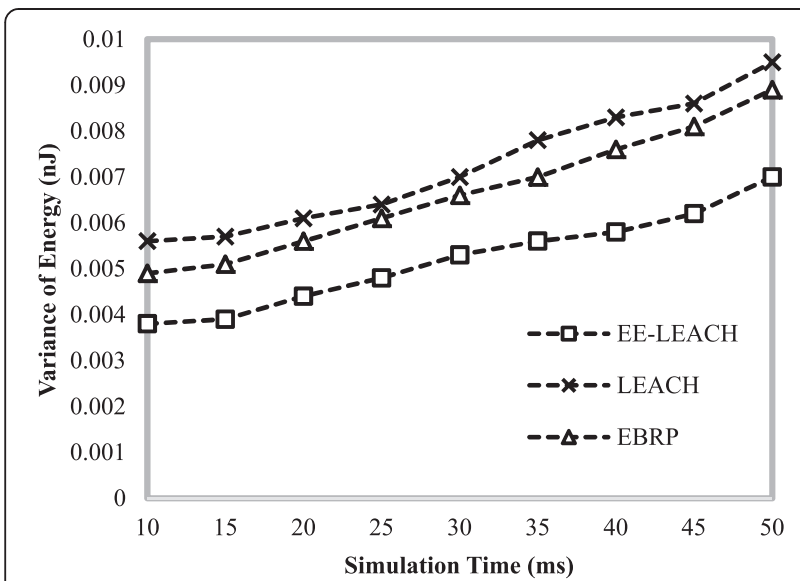

Figure 5 Variance of energy between EE-LEACH, EBRP and LEACH by varying the simulation time from 10 to $50 \mathrm{~ms}$.

forward the data to BS than the exiting EBRP and LEACH Protocols.

Packet delivery ratio (PDR) is the ratio of the number of delivered data to the BS. The greater value of PDR results in better performance of the system:

$$
P D R=\frac{\sum \text { Number of packet receive }}{\sum \text { Numeber of packet send }}
$$

Figure 8 shows the packet delivery ratio of the proposed EE-LEACH with the existing EBRP and LEACH Protocols. The proposed system results in a greater packet delivery ratio than the existing protocols.

From the above experimental results, it obviously proves that the proposed system can perform better than the existing protocol for data aggregation. Hence, the objective of the EE-LEACH is achieved.



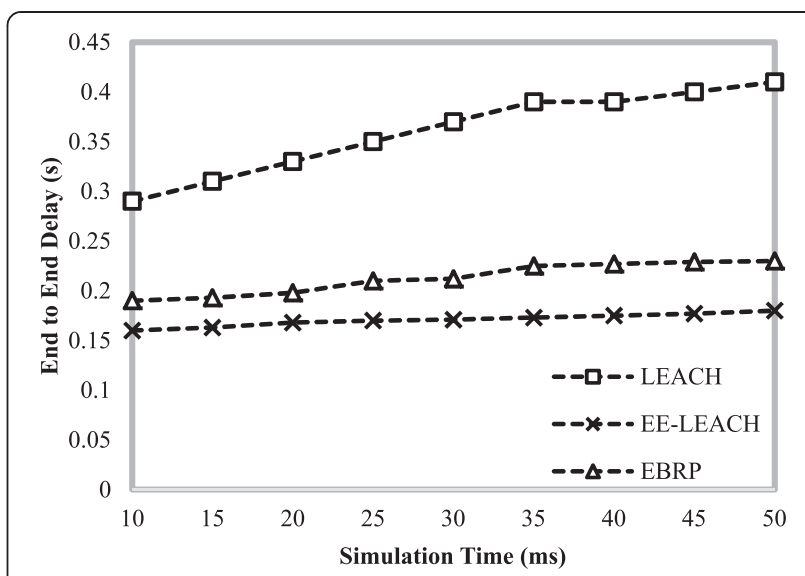

Figure 7 End-to-end delay for EE-LEACH with the existing EBRP and LEACH Protocols.

\section{Conclusion and future work}

In this paper, an energy-efficient LEACH Protocol is presented to improve the lifetime of the sensor network. The coverage probability is derived with respect to the Gaussian distribution. A sorting algorithm based on the residual energy of the neighbor nodes is executed to obtain the list of neighbor nodes. Data ensemble also takes place while aggregating the nodes. Data ensemble can save considerable energy while the source nodes forming one cluster are deployed in a relatively small area when the sink node is far away from the source nodes. An election weight is determined by taking account of the concentration degree of $\mathrm{SNs}$ and their residual energy for optimal $\mathrm{CH}$ election. The EE-LEACH Protocol results in a better packet delivery ratio, lesser energy consumption and lesser E2E delay than the EBRP and LEACH Protocols. The experimental results shows that the proposed EE-LEACH yields better outcomes than the existing EBRP and LEACH Protocols.

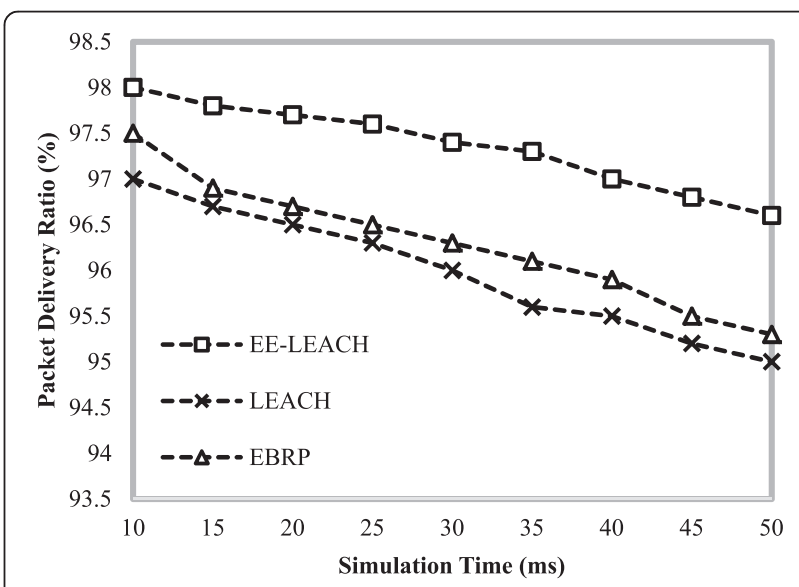

Figure 8 Packet delivery ratio for EE-LEACH with the existing EBRP and LEACH Protocols. 
This paper only focuses on reducing the energy consumption. But it lacks to provide the confidentiality and integrity of data. We extend this work with the security concepts, which analyses the traffic flow among the sensor nodes. Hence, in the future, the proposed EELEACH Protocol is integrated with the security mechanisms to protect the network from security attacks.

\section{Competing interests}

The authors declare that they have no competing interests.

\begin{abstract}
Authors' information
DR.A.GOPI SAMINATHAN was born on 23rd October 1973 in Dindigul, Tamilnadu, India. He pursued his Bachelor's Degree in Electronics and Communication Engineering from MK University, Madurai in the year 1995. He received his Master's Degree in Applied Electronics in the year 2006 from Anna University, Chennai and Ph.D., degree in the faculty of Information and Communication Engineering, Anna University, Chennai, Tamilnadu, India in the year 2013. Presently working as a Professor and Head in Department of ECE, SSMIET, Dindigul, Tamilnadu, India.

He started his career as an Assistant Professor in the year 1999. He has worked in various reputed colleges in Tamilnadu and has 16 years of service in teaching. He also holds 3 years of service in industry. His research interest includes Wireless Sensor Networks and Signal Processing. He is a life member of Indian Society for Technical Education (MISTE) and life time fellow of Institute of Electronics and Telecommunication Engineering (FIETE). He has published his research articles in nine international journals and presented many papers in National and International conferences. P.THIRUMURUGAN was born on 21th December 1977 in Oddanchatram, Tamilnadu, India. He received the Bachelor's Degree in Electronics and Communication Engineering from Anna University, Chennai in the year 2005. His Master's Degree is in Applied Electronics completed in the year 2007 from Anna University, Chennai, Tamilnadu, India. The present work is for earning Ph.D., degree in the faculty of Information and Communication Engineering, Anna University Chennai, Tamil Nadu, India. He worked as an Assitant Professor in the field of Electronics and Communication Engineering in RVS College of Engineering and Technology, Dindigul from 2007 to 2011. He is now working as an Assistant Professor in PSNA College of Engineering and Technology, Dindigul, Tamil Nadu, India. His research interest includes image processing in the denoising image. He is a life member of Indian Society for Technical Education (ISTE). He has published some of his research articles in the International journals and presented many papers in National and International conferences.
\end{abstract}

Received: 25 October 2014 Accepted: 21 February 2015

Published online: 20 March 2015

\section{References}

1. Y-h Zhu, W W-d, J Pan, T Y-p, An energy-efficient data gathering algorithm to prolong lifetime of wireless sensor networks. Comput. Commun. 33, 639-647 (2010)

2. S Gao, H Zhang, SK Das, Efficient data collection in wireless sensor networks with path-constrained mobile sinks. IEEE Trans. Mob. Comput. 10, 592-608 (2011)

3. MH Anisi, AH Abdullah, SA Razak, Energy-efficient data collection in wireless sensor networks. Wirel. Sens. Netw. 3, 329 (2011)

4. Y-F Chen, X-G Fan, B Xu, Cluster head optimization strategy for WSN based on LEACH. Comput Eng. 22, 026 (2011)

5. D Guo, L Xu, LEACH Clustering Routing Protocol for WSN, in Proceedings of the International Conference on Information Engineering and Applications (IEA) 2012. vol. 219, ed. by Z Zhong (Springer, London, 2013), pp. 153-160

6. Y Yao, Q Cao, AV Vasilakos, EDAL: an energy-efficient, delay-aware, and lifetime-balancing data collection protocol for wireless sensor networks, in 2013 IEEE 10th International Conference on Mobile Ad-Hoc and Sensor Systems (MASS), (China, 2013), pp. 182-190

7. K Han, J Luo, Y Liu, AV Vasilakos, Algorithm design for data communications in duty-cycled wireless sensor networks: a survey. IEEE Commun. Mag. 51, 107-113 (2013)

8. L Xiang, J Luo, A Vasilakos, Compressed data aggregation for energy efficient wireless sensor networks, in 2011 8th Annual IEEE Communications
Society Conference on Sensor, Mesh and Ad Hoc Communications and Networks (SECON), (Seattle, 2011), pp. 46-54

9. N Chilamkurti, S Zeadally, A Vasilakos, V Sharma, Cross-layer support for energy efficient routing in wireless sensor networks. J. Sens. 2009, 1-9 (2009)

10. G Wei, Y Ling, B Guo, B Xiao, AV Vasilakos, Prediction-based data aggregation in wireless sensor networks: combining grey model and Kalman filter. Comput Commun. 34, 793-802 (2011)

11. X-Y Liu, Y Zhu, L Kong, C Liu, Y Gu, AV Vasilakos, et al., CDC: compressive data collection for wireless sensor networks.

12. D Kumar, TC Aseri, R Patel, EECDA: energy efficient clustering and data aggregation protocol for heterogeneous wireless sensor networks. Int. J. Comput. Commun. Control 6, 113-124 (2011)

13. D Wei, Y Jin, S Vural, K Moessner, R Tafazolli, An energy-efficient clustering solution for wireless sensor networks. Wirel. Commun. IEEE Transac. 10, 3973-3983 (2011)

14. X Min, S Wei-Ren, J Chang-Jiang, Z Ying, Energy efficient clustering algorithm for maximizing lifetime of wireless sensor networks. AEU-Int J. Electron. Commun. 64, 289-298 (2010)

15. R Fengyuan, Z Jiao, H Tao, L Chuang, SKD Ren, EBRP: energy-balanced routing protocol for data gathering in wireless sensor networks. IEEE Transac. Parallel. Distrib. Syst. 22, 2108-2125 (2011)

16. D Karaboga, S Okdem, C Ozturk, Cluster based wireless sensor network routing using artificial bee colony algorithm. Wirel. Netw. 18, 847-860 (2012)

17. J Yuea, W Zhang, W Xiao, D Tang, J Tang, Energy efficient and balanced cluster-based data aggregation algorithm for wireless sensor networks. Procedia. Eng. 29, 2009-2015 (2012)

18. RR Rout, SK Ghosh, Adaptive data aggregation and energy efficiency using network coding in a clustered wireless sensor network: an analytical approach. Comput. Commun. 40, 65-75 (2014). 3/1

19. L Hui, H Uster, Exact and heuristic algorithms for data-gathering clusterbased wireless sensor network design problem. Netw. IEEE/ACM Transac 22, 903-916 (2014)

20. BS Mathapati, SR Patil, VD Mytri, Energy efficient reliable data aggregation technique for wireless sensor networks, in 2012 International Conference on Computing Sciences (ICCS), (Reykjavík, Iceland, 2012), pp. 153-158

21. JP Sheu, PK Sahoo, CH Su, WK Hu, Efficient path planning and data gathering protocols for the wireless sensor network. Comput. Commun. 33, 398-408 (2010). 2/26

22. D Ebrahimi, C Assi, Compressive data gathering using random projection for energy efficient wireless sensor networks. Ad Hoc Netw. 16, 105-119 (2014)

23. JK Min, CW Chung, EDGES: Efficient data gathering in sensor networks using temporal and spatial correlations. J. Syst. Softw. 83, 271-282 (2010). 2//

24. W Jin, T Shaojie, Y Baocai, L Xiang-Yang, Data gathering in wireless sensor networks through intelligent compressive sensing, in 2012 Proceedings IEEE INFOCOM, (Toronto, Canada), pp. 603-611, 2012

25. Y Song, L Liu, H Ma, AV Vasilako, A biology-based algorithm to minimal exposure problem of wireless sensor networks. IEEE Trans. Netw. Serv. Manag. 11, 417-430 (2014)

26. L Liu, Y Song, H Zhang, H Ma, A Vasilakos, Physarum optimization: a biology-inspired algorithm for the Steiner tree problem in networks. IEEE Trans. Comput. 64(3), 819-832 (2013)

27. P Li, S Guo, S Yu, AV Vasilakos, CodePipe: an opportunistic feeding and routing protocol for reliable multicast with pipelined network coding, in 2012 Proceedings IEEE INFOCOM, (2012), pp. 100-108.

28. Y Zeng, K Xiang, D Li, A Vasilakos, Directional routing and scheduling for green vehicular delay tolerant networks. Wire. Net. 19, 161-173 (2013). /02/01 2013 